\title{
Allergologie: Qualität vor Quantität
}

W ährend früher die Aufgaben einer wissenschaftlichen Fachgesellschaft sich in der regelmäßigen Organisation von Kongressen sowie in gelegentlichen Rundbriefen an die Mitglieder erschöpften, sind durch die zunehmend harten Auseinandersetzungen im berufspolitischen, aber auch allgemein- und wissenschaftspolitischen Umfeld sehr viel mehr und arbeitsintensivere Aufgaben zu erledigen. Die DGAI hat dieser Entwicklung Rechnung getragen und eine Geschäftsstelle eingerichtet (Frau Erika Ratzinger, Fax: 089/58 38 24). Wir hoffen damit den Kontakt zu den Mitgliedern, aber auch $\mathrm{zu}$ allen Interessierten zu verbessern.

Für die offizielle Kommunikation haben wir glücklicherweise das Allergo Journal. Auch unsere Zeitschrift ist von den Umwälzungen in den Häusern Bertelsmann und Springer betroffen; es hat erhebliche personelle Ve $\mathrm{r}$ ä $\mathrm{n} d \mathrm{e}$ rungen geben (siehe Impressum). Das letzte Heft war dünn, nicht durch mangelnden Vorrat an Original- oder Übersichtsarbeiten!

Ein „Allergo-Letter“ von zweifelhaftem Inhalt über unkonventionelle Methoden hat gegen den Willen der Herausgeber seinen Weg als Beilage gefunden; wir wurden belehrt, daß eine o ffizielle Beilage nicht verboten werden könne, es sei denn, sie verstoße gegen die guten Sitten. Die Herausgeber hielten diesen Fall für gegeben, wobei allerdings die Definition der ,,Sittlichkeit" im Bereich von Wissenschaft und Medizin noch weniger präzise ist als bei der freiwilligen Selbstkontrolle anderer Medien. Wir betrachten es als wesentliche Aufgabe, die Qualität unserer Disziplin zu sichern.

Wir freuen uns deshalb über neue Mitglieder im Herausgeberkollegium. Prof. Kurt Blaser (Davos) und Priv. Doz. Harald Renz (Berlin) werden für wissenschaftliche Qualität einstehen. Auch im Beirat (Advisory board) hat eine Verjüngung stattgefunden.
Zur Qualitätssicherung gehören auch Positionspapiere und Leitlinien, wie wir dies in diesem („Pseudo-allergische Reaktionen") und den nächsten Heften fortsetzen werden.

Wissenschaftliche Fachgesellschaften vertreten die Interessen von Ärzten und Patienten. Es geht darum, den Standard der Ve r s o rgung allerg i e k r a nker Menschen in Deutschland stetig und nachhaltig zu verbessern. Neben Förderung der Forschung und Initiativen zur primären und sekundären Prävention gehört dazu auch die Qualitätskontrolle der ärztlichen We i t e r b i ldung. Die DGAI hat eine Offensive zur Verbesserung der Weiterbildung im Bereich Allergologie gestartet, die unter dem Motto „Qualität vor Quantität“ dafür eintreten will, daß in Zukunft in Deutschland weitergebildete „Allerg ologen" dem internationalen Standard entsprechen bzw. besser sind!

Bis vor kurzem gab es in einzelnen Bundesländern noch die Praxis, mangels genügend weiterbildungsberechtigter Personen einfach jedem, der die Zusatzbezeichnung Allergologie erworben hatte, auch die We i t e r bil d u ng sermächtigung zu geben. Wollte man dies auf den Straßenverkehr übertragen, wäre jeder Führerscheinbesitzer automatisch Fahrlehrer! Die Qualifikation der Weiterbildungsermächtigten in Person und Institution soll in Zukunft besser definiert und kontrolliert werden.

Zusammen mit dem Ärzteverband Deutscher Allergologen (ÄDA) org anisieren wir regelmäßige Pressekonferenzen. Wir planen mit der „Deutschen Akademie für Allergologie und Um-

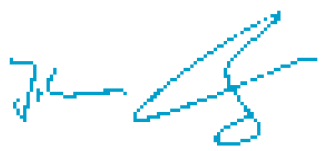

Prof. Dr. Dr. J. Ring weltmedizin“ (DAAU) in diesem Jahr die Erstellung eines Weißbuches „Alle rgie in Deutschland", das in kompakter Weise die wichtigsten wissenschaftlichen, klinischen und sozioökonomischen Fakten allgemein verständlich und mit praktischen Vo r s c h läg e n zur Umsetzung für alle Interessierten in Gesellschaft und Politik zusammenfassen soll.

Im Oktober (20. bis 24.10.1999) wird in München der große Deutsche Alle rgie-Kongreß stattfinden (zuletzt 1996 in Freiburg). Wir fordern alle Interessierten auf, sich rechtzeitig anzumelden und Beiträge einzureichen. Wi r werden dort neben den wissenschaftlichen und klinischen Fortschritten auch standespolitische Fragen diskutieren und den Dialog mit den Selbsthilfegruppen führen.

Wenn Sie an speziellen Fragestellungen in der Allergologie interessiert sind, lassen Sie uns dies bitte wissen oder machen Sie in einer der dafür eingerichteten Sektionen der DGAI (Pneumologie, Pädiatrie, Dermatologie, HNO, Umweltmedizin, Immunologie) mit.

Daß Allergien die Dimension einer „, Volkskrankheit" bereits haben und im kommenden Jahrtausend wahrscheinlich noch stärker haben werden, belegt eine Umfrage der DAK, die vom Präsidenten des ÄDA, Dr. Wenning, in diesem Heft vorgestellt und kommentiert wird.

Angesichts der anstehenden neuen Gesundheitsreform mit noch unklarem Ausgang müssen wir als kleines und interdisziplinär orientiertes Fachgebiet gewappnet sein und zusammenhalten!

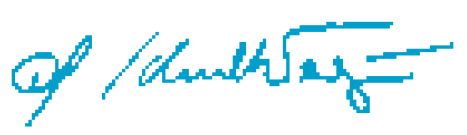

Prof. Dr. G. Schultze-Werninghaus 